\title{
In Silico Target Deconvolution of Curcumin (Diferuloylmethane) Against Rubella Virus (RUBV) and Hepatitis Delta Virus (HDV)
}

\author{
Mansi.J. Shah ${ }^{1}$, Roopa. $\mathrm{L}^{1^{*}}$ Pravin Kumar.R ${ }^{2}$ \\ ${ }^{I}$ msc Biotechnology, Mount Carmel College, Bangalore. \\ ${ }^{1 *}$ Lecturer, Department of Biotechnology, Mount Carmel College, Bangalore. \\ ${ }^{2}$ Senior Scientist, Polyclone Bioservices Pvt Ltd, Bangalore
}

\begin{abstract}
Recent studies have been done on the antiviral properties of Curcumin. In relation to this Curcumin has been docked against the proteome of the ssRNA(+) Rubella virus (RUBV) and ssRNA(-) Hepatitis D virus (HDV) to identify the proteins which may act as targets for curcumin. The docking studies show low binding energies of curcumin with Non-structural polyprotein of RUBV with $K_{i}$ value as low as $2.04 \mu M$ and Small delta antigen of $H D V$ with $K_{i}$ value $53.04 \mu M$. The study has identified the same proteins to be the susceptible target in RUBV and $H D V$ for binding of curcumin or its bioconjugates to combat the virus.
\end{abstract}

Keywords: Autodock, I-TASSER, Viral zone, Non-structural polyprotein, Small delta antigen

\section{Introduction}

Curcumin, commonly known as diferuloylmethane, is a natural yellow pigment derived from rhizomes of the plant Curcuma longa (Zingiberaceae), a plant grown in tropical Southeast Asian and Indian subcontinent. Studies on curcumin reveal that this compound has significant anti-inflammatory, antimicrobial, antioxidant, anticarcinogenic and more recently discovered antiviral properties ${ }^{1,2,3}$. Rubella, also known as German measles or three-day measles, is a disease caused by the rubella virus. Infection of the mother by Rubella virus within the first 20 weeks of pregnancy may be serious; the child may be born with congenital rubella syndrome (CRS), which entails a range of serious incurable illnesses ${ }^{4}$. The RUBV genome contains two long open reading frames (ORFs).The 5'-proximal ORF encodes nonstructural proteins (NSPs) primarily involved in viral RNA replication, while the 3'-proximal ORF extends encodes the virion structural proteins. The genome encodes 2 nonstructural polypeptides (p150 and p90) within its 5'-terminal two-thirds and 3 structural polypeptides (C, E2, and E1) within its $3^{\prime}$-terminal one-third. Both envelope proteins E1 and E2 are glycosylated ${ }^{5,6}$.Nonstucturalpolyprotein is reported to have a role in viral replication ${ }^{7}$. The genome sequence and the description of amino acid sequence motifs which are characteristic for helicase proteins have indicated that the polyprotein region located between amino acid residues 1300 and 1600 represents the Rubella virus helicase ${ }^{8}$. The structural proteinof RUBV includesa capsid protein that interacts with viral genomic RNA to form a nucleocapsid and two membrane glycoproteins, E2 and E1. The region of E1 between amino acids 81 and 109 is involved in the membrane fusion activity of RUBV and it may be important for the interaction of that protein with E2 to form the E1-E2 heterodimer?.

Hepatitis D Virus (HDV) classified as Hepatitis delta virus is a small circular enveloped ss(-)RNA virus $^{10}$.The diseases associated with this virus are Fulminant acute hepatitis, severe chronic active hepatitis, hepatocellular carcinoma ${ }^{11,12}$. HDV proteome consists of Large delta antigen and Small delta antigen as the envelope proteins ${ }^{13,14}$. The hepatitis delta virus large antigen (LHDAg) is a virally encoded protein that contains a prenylation signal sequence at its carboxyl terminus consisting of the tetrapeptideCys- Arg-Pro- Gln. It is reported that prenylation of LHDAg is required for the assembly of the hepatitis delta viral particle ${ }^{15}$. Leu-115 within the putative helix-loop-helix structure of the small HDAg is important for the replication of HDV but is not essential for RNA assembly ${ }^{16}$. HDV small delta antigen (S-HDAg) plays a critical role in virus replication. It has been reported that phosphorylation at serine 177 is a distinguishing feature of S-HDAg in the regulation of HDV RNA replication ${ }^{17,18}$. Small delta antigen of HDV (SHDAg) is an acetylated protein and mutation of SHDAg at Lys-72 modulates HDAg subcellular localization and may participate in viral RNA nucleocytoplasmic shuttling and replication ${ }^{19}$.

Curcumin is proven to bind directly to diverse proteins owing to its hydrophobic structure with the phenolic and the carbonyl functional groups located in the ends and the center of the molecule respectively. The structure enables curcumin not only to participate in hydrogen bonding with the targets, but also provides a strong electrostatic interaction to increase favorable free energies of association. According to a study on multitargeting by curcumin it is shown that curcumin binds to various proteins ${ }^{20}$. For this reason the proteome of RUBV and HDV were docked with curcumin and the mode of interactions were studied. 


\section{Methods}

The structure of RUBV Non-structural polyprotein and that of HDVLarge delta antigen were obtained from MODBASE and RUBV Structural polyprotein and HDV Small delta antigen were modelled using ITASSER $^{21,22,23}$. The tool employs eight different threading approaches such as MUSTER, HHSEARCH, PROSPECT2, PPA-I, FFASO3, SP3, SPARKS \& SAMT99 to align the protein sequence on the different templates. Also, top 10 templates were obtained on the basis of Structure Alignment using the program TM-Align. The structure with highest $\mathrm{C}$ - score (a confidence score for estimating the quality of predicted models by ITASSER), calculated based on the significance of threading template alignments and the convergence parameters of the structure assembly simulations. C-score values range between (0-1) where a higher score indicates a more reliable ligand binding site prediction. Any model with a TM score (Measure of global structural similarity between query and template) of more than 0.5 indicates a model of correct topology. 3D structure of the ligand was obtained from the chemical database ZINC. The receptors and ligand were prepared using MGL tools 1.5.6 and grids were prepared for all the proteins, the grid size and the grid points varied from protein to protein owing to the different sizes of each. The grid was set to encompass the complete protein which is a procedure for blind docking analysis. Docking studies were performed using AutoDock4.2.5.1. AutoDockis an automated procedure for predicting the interaction of ligands with biomacromolecular targets. Itconsists of two main programs: AutoDock for docking of the ligand to a set of grids describing the target protein and AutoGrid for pre-calculating these grids.

The Lamarckian Genetic Algorithm (LGA) search engine with empirical free energy function for estimation of free energy of binding, docking energy, inhibitory constant, intermolecular energy and torsional free energy were used for separate docking runs. The scoring function included the electrostatic energy, van der Waals energy, hydrogen bonding energy and the desolvation effect based on which the binding free energy were calculated.

The docking results of each protein was tabulated and each docked conformation was studied and compared based on binding energy, electrostatic energy and inhibitory constant to identify the possible target(s) of the ligand.

\subsection{Modelling of Structural Polyprotein of RUBV}

\section{Results of Modelling}

The protein sequence was aligned best with the $4 \mathrm{adgA}$ template. The percentage sequence identity of the whole template with the Structural polyprotein was 40\%, the Z- score for this alignment being 2.16. Table 1 shows the top three templates used by I-TASSER for alignment and modelling. Table 2 shows the top 3 templates on the basis of structural alignment, the best template being 1ej6C with TM-score of 0.876. Fig. 1 shows structure of the modelled Structural polyprotein along with the estimated accuracy of the model with TM Score being 0.69 \pm 0.12 .

\subsection{Modelling of Small Delta Antigen of HDV}

The submitted amino acid sequence of Small delta antigen aligned best with the template $3 \mathrm{ikmA}$. The percentage sequence identity of the whole template with the Small delta antigen was $23 \%$ and the Z- score for this alignment being 1.19. Table 4 shows the top three templates used by I-TASSER for alignment and modelling of Small delta antigen. Table 5 shows the top 3 templates on the basis of structural alignment, the best template being 4btgA1 with TM-score of 0.476.Fig. 2 shows the structure of modelled Small delta antigen along with the estimated accuracy of the model with TM Score being $0.44 \pm 0.14$.

\section{Results of Docking}

Table 7 shows the binding energy and inhibitory concentration of the RUBV and HDV proteins that were docked with curcumin. A graphical representation of the binding energy for RUBV and HDV proteins docked with curcumin is shown in Graph 1. In case of RUBV best results were observed for Non-structural polyproteinwith binding energy $-7.76 \mathrm{Kcal} / \mathrm{mol}$ and $\mathrm{K}_{\mathrm{i}}$ value of $2.04 \mu \mathrm{M}$ follwed by Structural polyprotein with binding energy $-5.50 \mathrm{Kcal} / \mathrm{mol}$ and $\mathrm{K}_{\mathrm{i}}$ value of $92.56 \mu \mathrm{M}$ and in case of HDV Small delta antigen showed the best results with binding energy of $-5.83 \mathrm{Kcal} / \mathrm{mol}$ and $\mathrm{K}_{\mathrm{i}}$ value of $53.04 \mu \mathrm{M}$ followed by Large delta antigen with binding energy $-2.48 \mathrm{Kcal} / \mathrm{mol}$ and $\mathrm{K}_{\mathrm{i}}$ value of $15.33 \mathrm{mM}$.As it is known that the Non-structural polyprotein of RUBVhas a role in viral replication which replicates the 40S (+) genomic RNA into (-) antigenomic RNA ${ }^{7}$ and that of Small delta antigen of HDV activates RNA replication, whereas the large form suppresses this process but is required for viral particle assembly ${ }^{17}$.

\section{Discussion}

In case of HIV-1 integrase curcumin binds to the catalytic core comprising of Asp 64, Thr 66, His 67, Glu 92, Thr 93, Asp 116, Ser 119, and Asn 120 and Lys 159. In the HIV-1 Protease the curcumin structure fits well into active site interacting with amino acids Asp 25, Gly 27, Asp 29, Asp 30 and Asp 29, Asp 30 of chain A 
and $\mathrm{B}$ respectively. These interactions suggest that extensive hydrogen bonding promoted by the O-hydroxyl structure and electrostatic interactions are important for both HIV -1 integrase and HIV-1 Protease interaction ${ }^{20}$.In our analysis it was observed that the RUBV Non-structural polyproteinamino acids such as Val 836,Ala 853,Ala 856,Asn 857,Ala 858,Leu 862,His 883, Gly884,Tyr 887, Thr 888, His 889 and Ile 890 are seen to interact by van der Waals force of attraction with curcumin and Ile 854, Thr 880, Pro 881, Gly 882residues show electrostatic interactions with curcumin.Interactions of curcumin with Small delta antigen revealed that one of the O-hydroxyl groups of curcumin showed hydrogen bond interaction with Gly 193. The enolic atom of curcumin showed hydrogen bond interaction with Gly65.Leu 155,Ala 162,Pro 169,Ser 170 and Phe 179 are seen to interact by van der Waals force of attraction with curcumin. Lys 63, Asp 64, Gly 65, Pro 163, Gly165, Gly 166, Phe 167 and Gly 193 show electrostatic interactions with curcumin. Interestingly both Ala 162 and Pro 163 happen to be the predicted binding site residues of Small delta antigen as per the I TASSER results. In Fig. 3 and Fig. 4 the residues circled pink are those involved in electrostatic interactions and those circled green are involved in van der Waals interactions. Blue surface indicates aromatic ring edges.

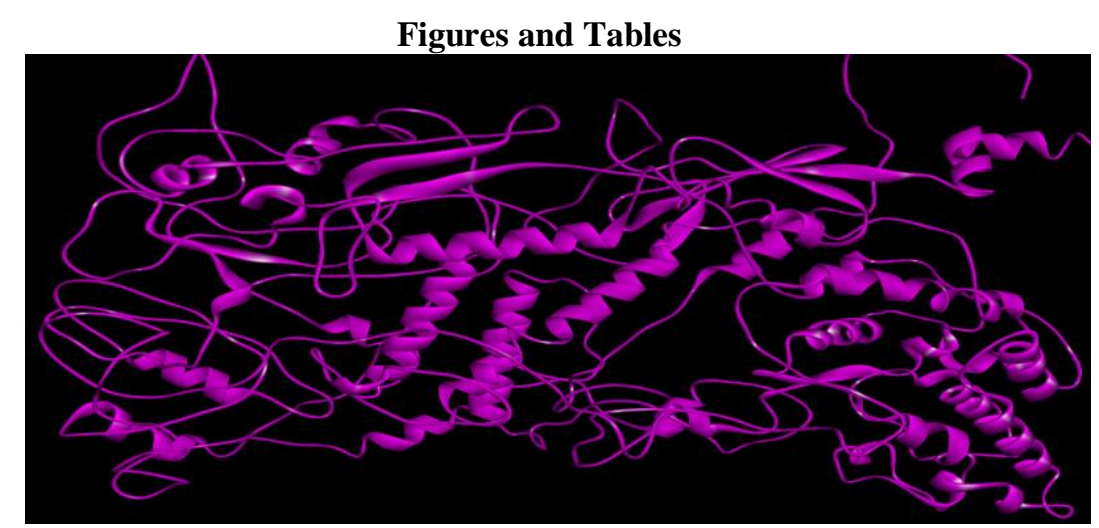

Fig1. Structure of Modelled Structural polyprotein (RUBV)

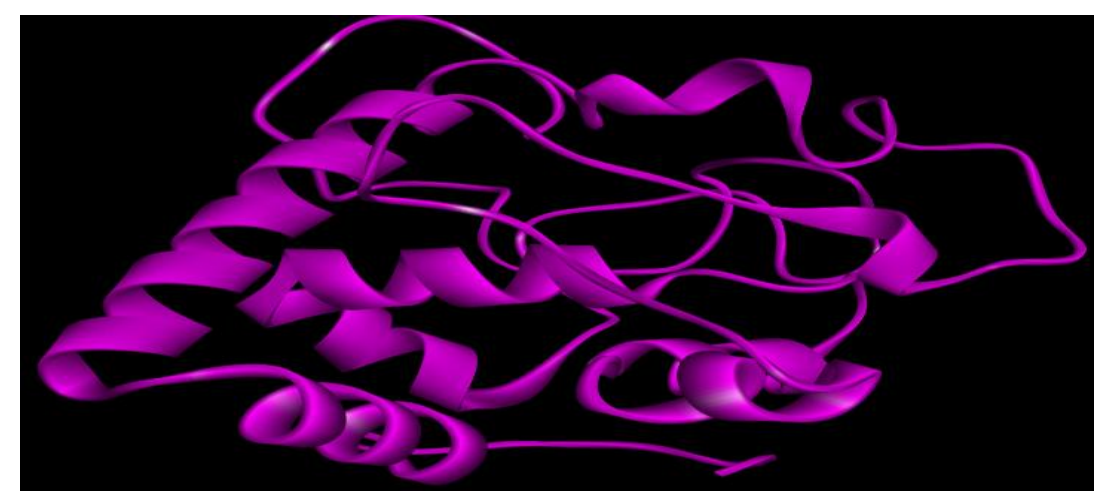

Fig2. Structure of Modelled Small delta antigen (HDV)

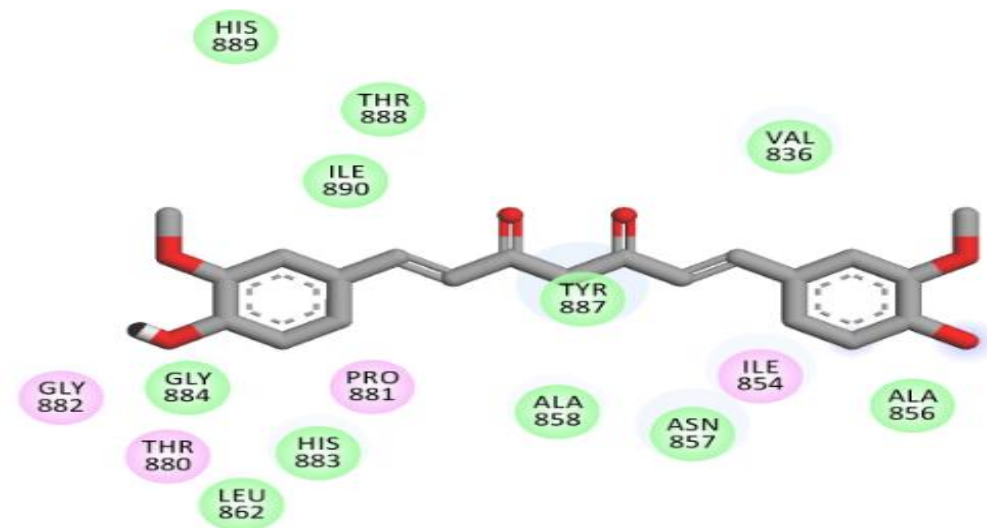

Fig3. Interactions of Curcumin with Non-structuralpolyprotein of RUBV 


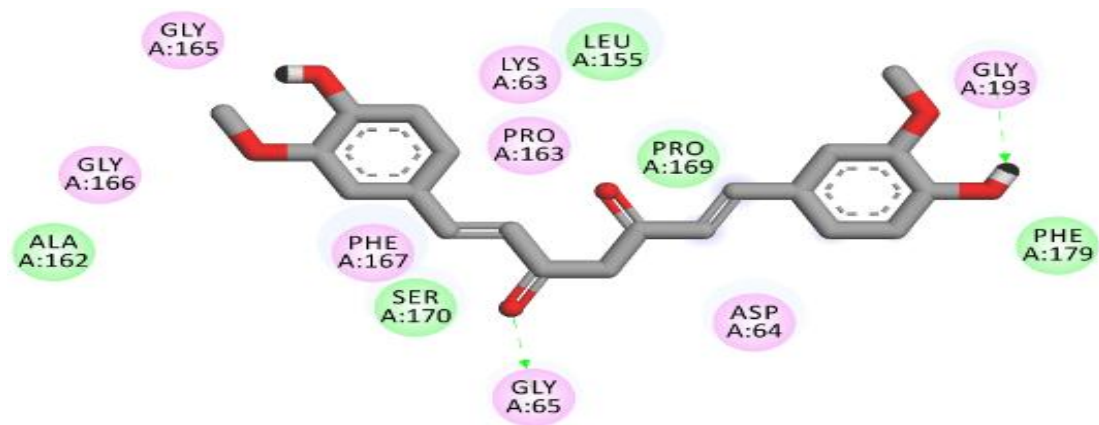

Fig4. Interactions of Curcumin with Small delta antigen of HDV

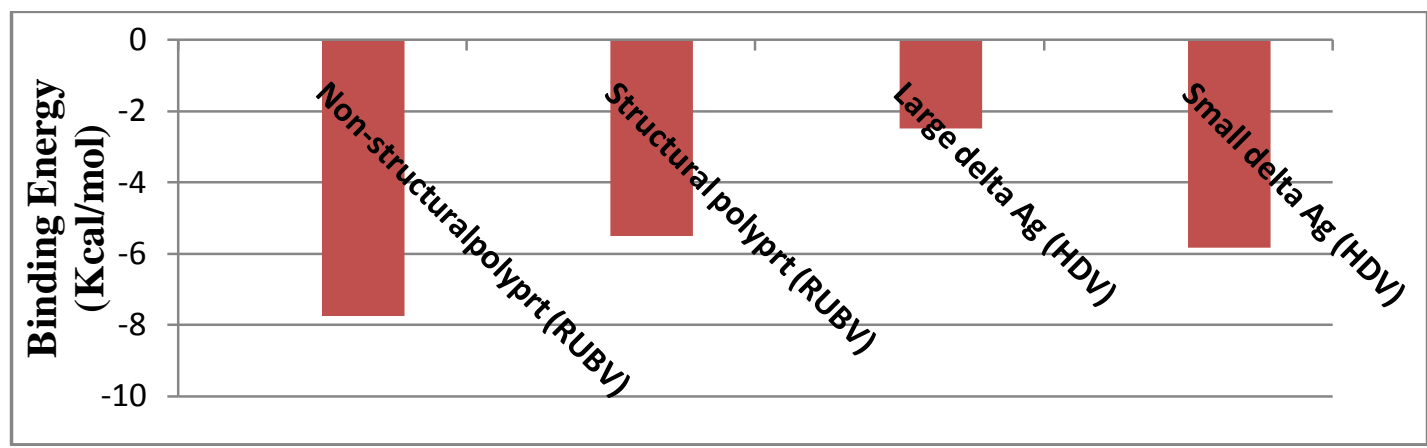

Graph 1: Binding Energy of the RUBV and HDV Proteins Docked with Curcumin

Table 1: Top 3 templates used by i-tasser for sequence alignment and modelling of structural polyprotein

\begin{tabular}{|l|l|l|l|l|}
\hline Rank & $\begin{array}{l}\text { PDB } \\
\text { Hit }\end{array}$ & $\begin{array}{l}\text { \% sequence identity of the template in } \\
\text { the threading aligned region with the } \\
\text { query sequence }\end{array}$ & $\begin{array}{l}\text { Coverage of } \\
\text { Thethreading } \\
\text { alignment }\end{array}$ & $\begin{array}{l}\text { Normalized Z Score } \\
\text { (Alignment with Normalized Z Score >1 } \\
\text { means a good alignment and vice versa) }\end{array}$ \\
\hline 1 & $4 \mathrm{adgA}$ & 0.99 & 0.41 & 2.16 \\
\hline 2 & $2 \mathrm{cseW}$ & 0.16 & 0.92 & 1.96 \\
\hline 3 & $2 \mathrm{gsxA}$ & 0.18 & 0.77 & 1.01 \\
\hline
\end{tabular}

Table 2: Top 3 Templates on the basis of Structural Alignment for Structural polyprotein

\begin{tabular}{|l|l|l|l|l|l|}
\hline Rank & PDB Hit & $\begin{array}{l}\text { TM } \\
\text { Score }\end{array}$ & $\begin{array}{l}\text { RMSD between the residues } \\
\text { that are structurally aligned by } \\
\text { TM - align }\end{array}$ & $\begin{array}{l}\text { \% sequence identity } \\
\text { in the structurally } \\
\text { aligned region }\end{array}$ & $\begin{array}{l}\text { Coverage of the } \\
\text { alignment by TM }- \text { align }\end{array}$ \\
\hline 1 & $1 \mathrm{ej} 6 \mathrm{C}$ & 0.876 & 2.59 & 0.133 & 0.910 \\
\hline 2 & $3 \mathrm{k} 1 \mathrm{gC}$ & 0.762 & 3.37 & 0.112 & 0.816 \\
\hline 3 & $3 \mathrm{izxB}$ & 0.631 & 5.40 & 0.086 & 0.756 \\
\hline
\end{tabular}

Table3: Predicted binding sites of structural polyproteinbased on templates with similar binding sites

\begin{tabular}{|l|l|l|l|l|}
\hline Rank & PDB Hit & $\begin{array}{l}\text { Cscore }^{\mathrm{LB}} \\
\text { (confidence score of predicted }_{\text {binding site) }}\end{array}$ & $\begin{array}{l}\text { IDEN } \\
(\% \text { sequence identity in the } \\
\text { structurally aligned region) }\end{array}$ & $\begin{array}{l}\text { Predicted binding site } \\
\text { residue number }\end{array}$ \\
\hline 1 & $2 \mathrm{zxgA}$ & 0.01 & 0.029 & $53,55,74$ \\
\hline 2 & $1 \mathrm{~m} 56 \mathrm{D}$ & 0.01 & 0.073 & $91,98,99,102$ \\
\hline 3 & $1 \mathrm{~m} 56 \mathrm{~J}$ & 0.01 & 0.071 & $91,95,99,102,103$ \\
\hline
\end{tabular}

Table 4: Top 3 Templates used by I-TASSER for Sequence Alignment \&Modelling of Small delta antigen

\begin{tabular}{|l|l|l|l|}
\hline Rank & $\begin{array}{l}\text { PDB } \\
\text { Hit }\end{array}$ & $\begin{array}{l}\text { \% sequence identity of the } \\
\text { template inthe threading aligned region wit } \\
\text { thequery sequence }\end{array}$ & $\begin{array}{l}\text { Normalized Z Score } \\
\text { (Alignment with NormalizedZ Score >1 means agoo } \\
\text { alignment and vice versa) }\end{array}$ \\
\hline 1 & $1 \mathrm{a} 92 \mathrm{~A}$ & 0.84 & 6.58 \\
\hline 2 & $3 \mathrm{ikmA}$ & 0.13 & 1.19 \\
\hline 3 & $4 \mathrm{btgA}$ & 0.10 & 1.12 \\
\hline
\end{tabular}


Table 5: Top 3 Templates on the basis of Structural Alignment for Small Delta antigen

\begin{tabular}{|l|l|l|l|l|l|}
\hline Rank & PDB Hit & $\begin{array}{l}\text { TM } \\
\text { Score }\end{array}$ & $\begin{array}{l}\text { RMSD between the residues } \\
\text { that are structurally aligned } \\
\text { by TM - align }\end{array}$ & $\begin{array}{l}\text { \% sequence identity } \\
\text { in se structurally } \\
\text { aligned region }\end{array}$ & $\begin{array}{l}\text { Coverage of the alignment } \\
\text { by TM - align }\end{array}$ \\
\hline 1 & $4 \mathrm{btgA}$ & 0.476 & 5.03 & 0.042 & 0.774 \\
\hline 2 & $2 \mathrm{f} 5 \mathrm{uA}$ & 0.447 & 5.63 & 0.063 & 0.779 \\
\hline 3 & $1 \mathrm{wkvB}$ & 0.437 & 5.13 & 0.073 & 0.697 \\
\hline
\end{tabular}

Table 6: Predicted Binding Sites of Small delta antigenBased on Templates with Similar Binding Site

\begin{tabular}{|l|l|l|l|l|}
\hline Rank & PDB Hit & $\begin{array}{l}\text { Cscore } \mathrm{LB}^{\mathrm{LB}} \\
\text { (confidence score of predicted } \\
\text { binding site) }\end{array}$ & $\begin{array}{l}\mathrm{IDEN}^{\mathrm{a}} \\
(\boldsymbol{\%} \text { sequence identity in the } \\
\text { structurally aligned region) }\end{array}$ & $\begin{array}{l}\text { Predicted binding site } \\
\text { residue number }\end{array}$ \\
\hline 1 & $1 \mathrm{a} 50 \mathrm{~B}$ & 0.01 & 0.064 & $150,173,175$ \\
\hline 2 & $1 \mathrm{c} 29 \mathrm{~B}$ & 0.01 & 0.081 & $119,161,163$ \\
\hline 3 & 1 fuyB & 0.01 & 0.071 & $120,112,117,131,146$ \\
\hline
\end{tabular}

Table7: Binding Energy and Inhibitory Concentration of the RUBV Proteins and HDV Proteins docked with

\begin{tabular}{|l|c|c|}
\hline \multicolumn{1}{|c|}{ Purcumin } & $\mathbf{K}_{\mathbf{i}}$ \\
\hline Pon-structural polyprotein (RUBV) & $\begin{array}{l}\text { Binding Energy } \\
\text { (Kcal/mol) }\end{array}$ & -7.76 \\
\hline Structural polyprotein (RUBV) & -5.50 & $92.04 \mu \mathrm{M}$ \\
\hline Large delta antigen (HDV) & -2.48 & $15.33 \mathrm{MM}$ \\
\hline Small delta antigen (HDV) & -5.83 & $53.04 \mu \mathrm{M}$ \\
\hline
\end{tabular}

\section{Conclusion}

Analyzing all the docked conformations of curcumin in Non-structural polyprotein of RUBV it was observed that curcumin binds to a pocket that is chiefly made of amino acids like Asn, Thr, His, Gly and that of Small delta antigen of HDV is made of amino acids Gly, Ser, Lys, Asp which is more of charged and hydrophilic residues. Moreover, the overall interaction of curcumin with Non-structural polyprotein of RUBV shows a lot of van der Waals force of attraction whereas that of HDV Small delta antigen shows a lot of electrostatic interactions and hydrogen bonds seem to align with the interactions reported for HIV 1 integrase and HIV 1 protease ${ }^{20}$, indicating that the possible target of curcumin in RUBV is Non-structural polyprotein and in HDV is Small delta antigen. Having known the mode of interaction of curcmin with Non-structural polyprotein of RUBV and Small delta antigen of HDV,curcminbioconjugates with better bioavailability and lower inhibitory concentrations may be designed to interact fight the complications of RUBV and HDV.

\section{Acknowledgement}

We would like to acknowledge and extend our heartfelt gratitude to Dr. Preetha Nair for her constant support and encouragement. We thank Sr. Juanita, Principal, Mount Carmel College, for permitting us to use the laboratory facilities to complete the work.

\section{References}

[1]. Aggarwal BB, Kumar A, Bharti AC: Anticancer potential of curcumin: preclinical and clinical studies. Anticancer Res 2003, 23:363-98.

[2]. Ramendra K. Singha, , DiwakarRai a , DiptiYadava , A. Bhargava b , J. Balzarini c , E. De Clercqc: Synthesis, antibacterial and antiviral properties of curcumin bioconjugates bearing dipeptide, fatty acids and folic acid. European Journal of Medicinal Chemistry 45 (2010) 1078-1086.

[3]. Preetha, Anand., Sherin G, Thomas., Ajaikumar B, Kunnumakkara., Chitra, Sundaram., Kuzhuvelil B, Harikumar., Bokyung, Sung., Sheeja T, Tharakan., Krishna, Misra.,Indira K, Priyadarsini., Kallikat N, Rajasekharan., Bharat B, Aggarwal.. Biological activities of curcumin and its analogues (Congeners) made by man and Mother Nature. Biochemical pharmacology 76, 2008, 1590 - 1611.

[4]. Siegel M, Fuerst HT, Guinee VF (1971). "Rubella epidemicity and embryopathy. Results of a long-term prospective study". Am. J. Dis. Child.121 (6): 469-73. PMID5581012.

[5]. Chen J.,JH. Strauss, and TK Frey:Characterization of rubella virus non structural protease domains and its cleavage site. Virol,1996;76:4707-4713.

[6]. Dominguez G, Wang CY, Frey TK: Sequence of the genome RNA of rubella virus: evidence for genetic rearrangement during togavirus evolution. Virology, July 1990;177 (1): 225-38.

[7]. Xiu Liu, Susan C.Ropp, Richard J. Jackson, Jeryl K. Frey:The rubella virus non structural protease requries divalent cations for activity and function in trans.Journal of Virology,May 1998,p.4463-4466.

[8]. Gros C, Wengler G.: Identification of an RNA-stimulatedNTPase in the predictedhelicasesequence of the Rubella virusnonstructuralpolyprotein. Virology,1996 Mar 1;217(1):367-72.

[9]. Yang D, Hwang D, Qiu Z, Gillam S. Effects of mutations in the rubella virus E1 glycoprotein on E1-E2 interaction and membrane fusion activity. Journal of Virology. 1998 Nov; 72(11):8747-55

[10]. Makino S, Chang MF, Shieh CK, Kamahora T, Vannier DM, Govindarajan S, Lai MM: Molecular cloning and sequencing of a human hepatitis delta (delta) virus RNA. Nature. 1987 Sep 24-30; 329(6137):343-6. 
[11]. Rizzetto M, Ciancio A.: Epidemiology of hepatitis D. Seminars in Liver Disease. 2012 Aug; 32(3):211-9.

[12]. Fattovich G, Giustina G, Christensen E, Pantalena M, Zagni I, Realdi G, Schalm SW: Influence of hepatitis delta virus infection on morbidity and mortality in compensatedcirrhosistypeB. The European Concerted Action on Viral Hepatitis (Eurohep). Gut. 2000 Mar; 46(3):420-6.

[13]. Poisson F, Roingeard P, Baillou A, Dubois F, Bonelli F, Calogero RA, Goudeau A: Characterization of RNA-bindingdomains of hepatitisdelta antigen. The Journal of general Virology. 1993 Nov; 74 ( Pt 11):2473-8.

[14]. Saldanha JA, Thomas HC, Monjardino JP: Cloning and sequencing of RNA of hepatitis delta virus isolated from human serum. The Journal of general Virology. $1990 \mathrm{Jul} ; 71$ (Pt 7):1603-

[15]. James C. Otto and Patrick J. Casey: The hepatitis delta virus large antigen is farnesylated both in vitro and in animal cells. The Journal of Biological Chemistry. 1996 Mar 1; 271(9):4569-72.

[16]. Ming-Fu Chang, Chih-Hsin Chen, Syh-Lu Lin, Chun-Jung Chen,and Shin C. Chang. Functional Domains of Delta Antigens and Viral RNA required for RNA Packaging of Hepatitis Delta Virus. Journal of Virology, Apr. 1995, P. 2508-2514

[17]. Yeh TS, Lo SJ, Chen PJ, Lee YH.: Casein kinase II and protein kinase Cmodulatehepatitis delta virus RNA replication but not empty viral particle assembly. Journal of Virology. 1996 Sep; 70(9):6190-8.

[18]. Jung-Jung Mu, 1 Ding-Shinn Chen,2 And Pei-Jer Chen.: The conservedserine177 in the deltaantigen of hepatitisdelta virus is one putative phosphorylation site and is required for efficient viral RNA replication. Journal of Virology. 2001 Oct; 75(19):9087-95.

[19]. Mu JJ, Tsay YG, Juan LJ, Fu TF, Huang WH, Chen DS, Chen PJ.: The small delta antigen of hepatitis delta virus is an acetylated protein and acetylation of lysine 72 may influence its cellular localization and viral RNA synthesis. Virology. 2004 Feb 5; 319(1):60-70.

[20]. Subash C, Gupta., Sahdeo, Prasad., JiHye, Kim., Sridevi, Patchva., Lauren J, Webb., Indira K, Priyadarsini., and Bharat B, Aggarwal. Multitargeting by Curcumin As Revealed By Molecular Interaction Studies. Natural Product Reports 28, 2011, 19371955.

[21]. Ambrish Roy, AlperKucukural, Yang Zhang. I-TASSER: a unified platform for automated proteinstructure and function prediction. Nature Protocols, vol 5, 2010, 725-738

[22]. Yang Zhang. I-TASSER server for protein 3D structure prediction. BMC Bioinformatics, 9, 2008, 40.

[23]. Ambrish Roy, Jianyi Yang \& Yang Zhang. COFACTOR: an accurate comparative algorithm for structure-based protein function annotation. Nucleic Acids Research, vol 40, 2012, W471-W477 